\title{
Blood-Oxygenation-Level-Dependent-(BOLD-) Based R2' MRI Study in Monkey Model of Reversible Middle Cerebral Artery Occlusion
}

\author{
Jing Zhang, Ying-min Chen, and Yun-ting Zhang \\ Department of Radiology, General Hospital, Tianjin Medical University, 154 Anshan Road Heping District Tianjin 300052, China \\ Correspondence should be addressed to Yun-ting Zhang, cjr.zhangyunting@vip.163.com
}

Received 10 September 2010; Revised 25 November 2010; Accepted 30 December 2010

Academic Editor: Andrea Vecchione

Copyright ( 2011 Jing Zhang et al. This is an open access article distributed under the Creative Commons Attribution License, which permits unrestricted use, distribution, and reproduction in any medium, provided the original work is properly cited.

\begin{abstract}
Objective. To investigate the value of BOLD-based reversible transverse relaxation rate (R2') MRI in detecting ischemic penumbra (IP) in a monkey model of reversible middle cerebral artery occlusion (MCAO) and time evolution of relative R2' (rR2') in infarcted core, IP, and oligemia. Materials and Methods. 6 monkeys were used to make MCAO by the microcatheter method. MR scans were performed at $0 \mathrm{~h}(1 \mathrm{~h}$ after MCAO), $1 \mathrm{~h}, 3 \mathrm{~h}, 6 \mathrm{~h}, 12 \mathrm{~h}, 24 \mathrm{~h}$, and $48 \mathrm{~h}$ after reperfusion. R2' was calculated using quantitative $\mathrm{T} 2$ and T2* maps. Ischemic area was subdivided into infracted core, IP and oligemia. rR2' was calculated respectively. Results. Reversible MCAO model for 4/6 monkeys was made successfully. rR2' values were significantly different at each time point, being highest in oligemia followed by IP and infarcted core $(P<.05)$. With reperfusion time evolution, $\mathrm{rR} 2^{\prime}$ in infarcted core showed a decreased trend: sharply decreased within 6 hours and maintained at 0 during $6-48$ hours $(P<.05)$. rR2' values in IP and oligemia showed similar increased trend: sharply increased within 6 hours, maintained a plateau during 6-24 hours, and slightly increased until 48 hours. Conclusion. BOLD-based R2' MRI can be used to describe changes of cerebral oxygen extract in acute ischemic stroke, and it can provide additional information in detecting IP. The time evolution rR2' in infarcted core, IP, and oligemia is in accordance with the underlying pathophysiology.
\end{abstract}

\section{Introduction}

The mismatch model between MRI perfusion-weighted imaging (PWI) and diffusion-weighted imaging (DWI) has been suggested to provide an estimation of real ischemic penumbra (IP) and is used as referring criteria to select patients who are within the first 6 hours after stroke onset and might benefit from thrombolysis [1-3]. However, recent studies indicated that the mismatch region is only a weak approximation of IP [4]. PWI cannot reliably discriminate between benign oligemia and penumbra, and DWI lesions do not always indicate the irreversible core of infarction [5]. It is well established that PET is a more accurate imaging modality to detect IP, which has decreased cerebral blood flow (CBF) but elevated oxygen extraction fraction (OEF), reflecting a compensatory mechanism in still-viable but threatened tissue [4-6]. However, PET is not suitable for emergency imaging study of acute cerebral infarction because of its limited availability and cost effectiveness.
The deoxyhemoglobin (deoxy-Hb) in cerebral capillaries and veins is an indicator of OEF, which can be visualized by T2-and T2*-based blood-oxygen-level-dependant (BOLD) MRI [7]. The potential for detecting focally increased OEF as reduced $\mathrm{T}^{*}$ signal was first reported by Roussel et al. [8] in rats middle cerebral artery occlusion (MCAO) model in 1995. Tamura et al. [7] also reported signal loss on T2* WI obtained before arrival of contrast medium on PWI in patients with acute ischemic stroke, potentially reflecting a high OEF in 2002. Similar MRI findings were also reported by Wardlaw and Von Heijne [9] in 2006 and Morita et al. [10] in 2008, respectively. However, the relationship between T2* and OEF is still controversial. In a small series of 5 patients, Donswijk et al. [11] found, despite of all patients showing increased OEF on PET, no significant relationship between $\mathrm{OEF}$ and $\mathrm{T}_{2}^{*}$ signal obtained before arrival of contrast medium either within or between subjects. This may be due to the fact that $\mathrm{T} 2 *$ is sensitive to large variations of the static magnetic field $(\Delta \mathrm{B} 0)$. $\mathrm{R} 2^{\prime}$ is referred to as reversible 
transverse relaxation rate, a derivational parameter from $\mathrm{T} 2 *$ and $\mathrm{T} 2\left(1 / \mathrm{T} 2^{\prime}=1 / \mathrm{T} 2 *-1 / \mathrm{T} 2, \mathrm{R} 2 *=1 / \mathrm{T} 2 *, \mathrm{R} 2=1 / \mathrm{T} 2\right.$, $\left.\mathrm{R} 2^{\prime}=1 / \mathrm{T} 2^{\prime}\right)[12,13]$. The use of $\mathrm{R} 2^{\prime}$ is reasonable because it is isolated from the influences of deoxy-Hb on $\mathrm{T}^{*}$ values and water content on T2 values. Several studies have used $\mathrm{T} 2$ ' to predict lesion growth in acute ischemic stroke and demonstrated that it is sensitive to local content of deoxy-Hb $[12,14,15]$.

The purposes of this study were to investigate: (1) the value of R2' in detecting IP in a monkey model of reversible MCAO; (2) time evolution of relative R2' (rR2') in infarcted core, IP, and oligemia.

\section{Materials and Methods}

2.1. Monkey Model of Reversible MCAO. This study was approved by the experimental animal ethical committee of our university. Six male monkeys (Macaca mulatta) with a mean weight of $8.6 \mathrm{~kg}$ (range: from 8 to $10 \mathrm{~kg}$ ) were supplied by Experimental Animal Center of Beijin Military Medical Academy. All animals were fasted for 12-15 hours before experiment. Anesthesia was induced by ketamine $(0.25 \mathrm{mg}$ intramuscular) and atropine $(8-10 \mathrm{mg} / \mathrm{kg}$ intramuscular) followed by $100 \mathrm{mg}$ ketamine and $50 \mathrm{mg}$ diazepam in $500 \mathrm{~mL}$ NS intravenous drip. Physiological parameters such as respiration, heart rate, body temperature, and blood pressure were monitored.

The MCAO model was established by an interventional microcatheter method as described by De Grespigny et al. [16]. A one-inch incision was made in right femoral groove, and the femoral artery and vein were isolated. The right femoral artery was punctured and a 5 French catheter sheath was placed in. A 5 French catheter was introduced over a guide wire and navigated under fluoroscopy in one common carotid artery in close proximity to the internal carotid artery. A 1.7 French microcatheter (Prowler 10 with two markers, Cordis Corporation, $0.55 \mathrm{~mm}$ outside dia-meter) was introduced over a transcend microwire (Cordis). The microcatheter and the microguide wire were navigated into a selected branch of MCA and advanced into a wedged position. The animals were maintained on a slow, continuous, heparinized drip infusion (100 U/hour) during the ischemia period. After one hour of ischemia, the microguide wire was removed and the animal was transported into MRI scanner. If cerebral ischemia was confirmed by DWI, the microcatheter was also removed, followed by infusion of 250,000 units of urokinase in $50 \mathrm{~mL}$ NS with a high-pressure injector $(2 \mathrm{~mL} / \mathrm{min})$. Effective reperfusion was confirmed by angiograms.

The criterions of a successful animal model included (1) the selected branch of MAC was not visualized on angiograms; (2) MR scan showed high signal intensity on DWI in the territory of selected branch of MCA after one-hour ischemia; (3) Postmortem specimen showed nonstained infarcted area on 2,3,5-triphenyltetrazolium chloride (TTC) staining.

2.2. Imaging Study. MRI scan was performed on a $1.5 \mathrm{~T}$ superconductive scanner (GE Twin Speed Infinity with Excite
I) with birdcage head 8-channel phase array coil. All animals were scanned at 0 hour ( 1 hour after MCAO) and 1, 3, 6, 12, 24 , and 48 hours after reperfusion. Slice thickness was $5 \mathrm{~mm}$ and FOV was $15 \mathrm{~cm} \times 15 \mathrm{~cm}$. Scanning protocol included, (1) T2WI (fast recovery FSE, TR $9002 \mathrm{~ms}$, TE $124 \mathrm{~ms}$, NEX 2, matrix $256 \times 256)$. (2) DWI (SE-EPI, b values of 0 and $1000 \mathrm{~s} / \mathrm{mm}^{2}$, TR $3000 \mathrm{~ms}$, TE $112.6 \mathrm{~ms}$, NEX 2, matrix $128 \times$ 128) (3) Quantitative T2-map[qT2] (4-echo SE, TR $1200 \mathrm{~ms}$, TE 25, 50, 75, $100 \mathrm{~ms}$; flip angle $150^{\circ}$, matrix $\left.256 \times 256\right)$ and Quantitative T2*-map[qT2*] (8-echo GRE, TR $150 \mathrm{~ms}$, TE $3.6,9.4,15.3,21.2,27.1,33.0,37.5,43.2 \mathrm{~ms}$, flip angle $24^{\circ}$, matrix $256 \times 256)(4)$ PWI (single-shot gradient recall echo planner imaging T2* WI, TR $2000 \mathrm{~ms}$, TE $80 \mathrm{~ms}$, flip of angle $90^{\circ}$, matrix $128 \times 128$, NEX 1, Gd-DTPA $0.1 \mathrm{mmol} / \mathrm{kg}$, inject rate $2 \mathrm{~mL} / \mathrm{s}$ ).

qT2-map and $\mathrm{qT}^{*}$-map were calculated according to the following formula: $\mathrm{SI}(\mathrm{t})=\mathrm{SI}_{0} \mathrm{e}^{-\mathrm{t} / \mathrm{T} 2}$ and $\mathrm{SI}(\mathrm{t})=$ $\mathrm{SI}_{0} \mathrm{e}^{-\mathrm{t} / \mathrm{T} 2^{*}}$. $\mathrm{qT} 2-\mathrm{map}$ is linked to $\mathrm{qT}^{*}$-map, resulting in $\mathrm{R} 2^{\prime}$-based BOLD imaging as follows: $1 / \mathrm{T} 2^{\prime}=1 / \mathrm{T} 2 *-1 / \mathrm{T} 2$, $\mathrm{R}^{*}=1 / \mathrm{T} 2 *, \mathrm{R} 2=1 / \mathrm{T} 2, \mathrm{R} 2^{\prime}=1 / \mathrm{T} 2^{\prime} . \mathrm{R} 2 *$ is total transverse relaxation rate; $\mathrm{R} 2$ is irreversible transverse relaxation rate; $\mathrm{R} 2^{\prime}$ is reversible transverse relaxation rate with the unit of $\mathrm{Hz}[12,13]$.

2.3. Postmortem and Pathological Studies. At 48 hours after reperfusion, all animals were killed by an overdose of pentobarbital just after MRI scan. The brains were put into a $-20^{\circ} \mathrm{C}$ refrigerator for an hour to make the specimens hardened. The brains were sliced into 5-mm axial slabs by using a home-made instrument and stained with 2,3,5triphenyltetrazolium chloride (TTC) in $37^{\circ} \mathrm{C}$ water bath for 30 minutes. The stained sections were recorded with a digital camera.

2.4. Imaging Analysis and Statistics. Lesion volumes were calculated on DWI at 0 hour (0-DWI), T2WI at 48 hours after reperfusion (48-T2WI)- and TTC-stained sections by integrating products of lesion area and slice thickness on each lesion-containing slice. Lesion volume percentage (LVP) was calculated according to formula: LVP = lesion volumes/bilateral hemispheres volumes $\times 100 \%$.

Ischemic brain tissues were classified into 3 subtypes: infarcted core (hyperintensive areas on both 0 -DWI and 48T2WI), IP (hyperintensive areas on 0 -DWI but iso on 48T2WI), oligemia (delayed mean transit time areas on PWI at 0 hour [0-MTT] but iso on both 0 -DWI and $48-\mathrm{T} 2 \mathrm{WI}$ ) (Figures 1, 2, 3, and 4).

Imaging registration was performed on an off-line workstation. The platform was Matlab 7.0, Windows XP SP2 with 3.0 GHz Intel Pentium $4 \mathrm{CPU}$ and $2 \mathrm{G}$ RAM. 0-DWI, 0MTT, and 48-T2WI were coregistered to R2' images of each time point using a free form deformation method [17].

5 region-of-interests (ROIs) were put into each subtype brain tissue, respectively. Each ROI was $10 \mathrm{~mm}^{2}$ in size. Relative R2' (rR2') was calculated according to the ratio of lesion and its minor area on contralateral healthy hemisphere.

Statistic analysis was performed with SPSS15.0 software. Paired $t$-test and correlation analysis were used for comparison of LVP. rR2' values in three-subtype brain regions at 


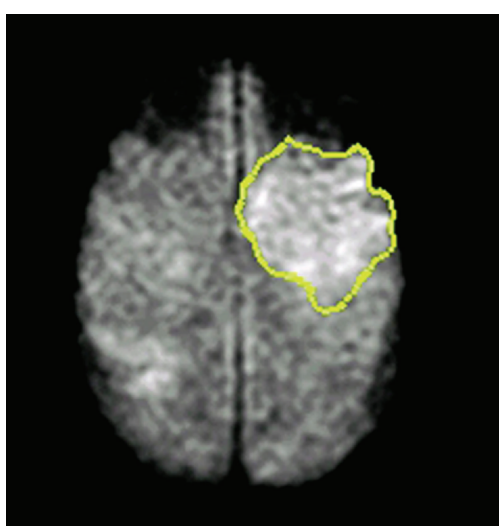

FIGURE 1: $0 \mathrm{~h}$ after MCAO DWI shows high-signal ischemic lesion.

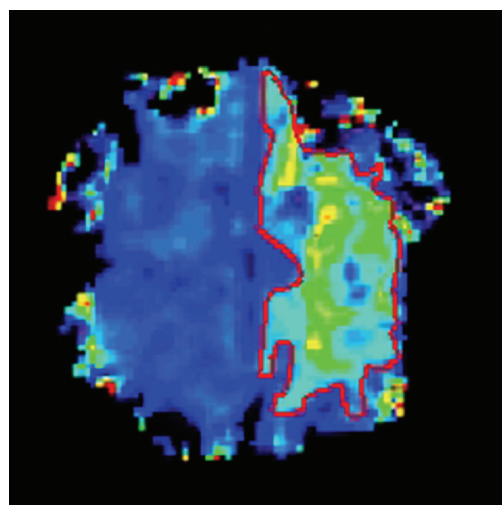

Figure 2: $0 \mathrm{~h}$ after MCAO PWI (MTT) shows delayed MTT lesion.

each time point were compared by ANOVA. Time evolution of $\mathrm{rR}^{\prime}$ in each subtype brain region was also compared by ANOVA. Statistic significance was $P<.05$.

\section{Results}

3.1. MCAO Model. MCAO model was successful in all 6 monkeys. 2 monkeys were dead at 2 and 14 hours after reperfusion because of large area of hemorrhagic infarction, respectively, and their data were not included in this study.

3.2. $L V P$. $0 \mathrm{~h}$ DWI demonstrated high-signal ischemic lesion and partial lesion can recover after reperfusion (Figures 5, 6, and 7), which was confirmed by TTC staining (Figure 8).

LVP on 0-DWI, 48-T2WI- and TTC-stained sections were summarized in Table 1. LVP on 48-T2WI was decreased compared with that on 0 -DWI $(t=6.472, P<.05$, ranged: $23 \% \sim 35 \%$, average $28 \%$ ). There was no statistic significant between LVP on 48-T2WI and that on TTC $(t=0.15, P>$ $.05)$, which showed significant positive correlation $(r=0.98$, $P<.05)$.

3.3. $r R 2^{\prime}$ in Different Ischemic Brain Tissue. On 0 hour R2' imaging, IP and oligemia showed hyperintensity compared to infarcted core. After reperfusion, the signal intensity on

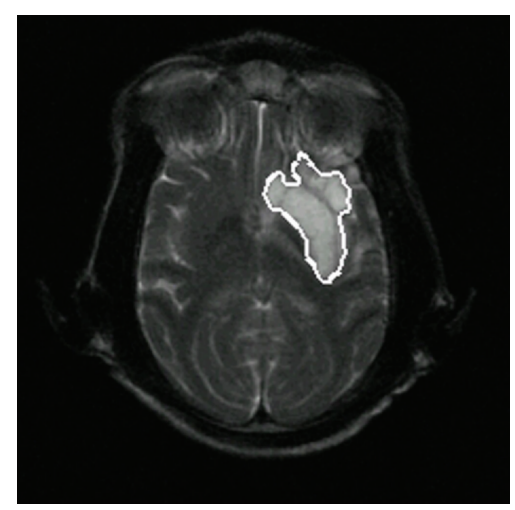

Figure 3: $48 \mathrm{~h}$ reperfusion T2WI shows high-signal infarction smaller than lesion on DWI.

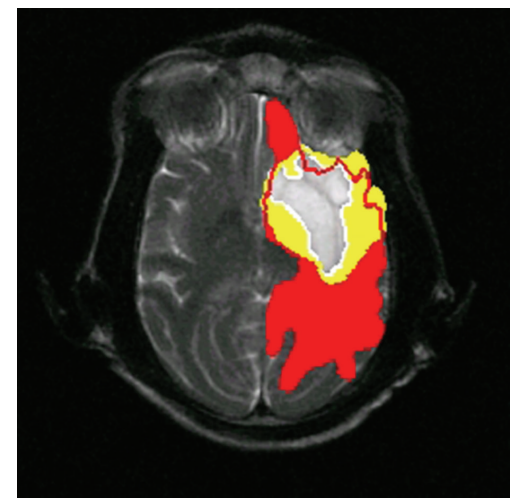

FIGURE 4: Overlapped imaging shows infarcted core (white), IP (yellow), and oligemia (red).

TABLE 1: LVP on DWI at 0 hour, T2WI at 48 hours after reperfusion and TTC.

\begin{tabular}{lccc}
\hline Animal number & DWI $(\%)$ & T2WI $(\%)$ & TTC $(\%)$ \\
\hline 1 & 10.77 & 8.08 & 8.19 \\
2 & 12.78 & 8.31 & 8.45 \\
3 & 12.26 & 8.77 & 8.68 \\
5 & 9.68 & 7.48 & 7.27 \\
\hline
\end{tabular}

$\mathrm{R} 2$ ' imaging was increased in IP and oligemia but decreased in infracted core with time evolution (Figures 9, 10, and 11). rR2' values of infracted core, IP, and oligemia at each time point after reperfusion were summarized in Table 2. rR2' values were significantly different at each time point, being highest in oligemia followed by IP and infracted core $(P<.05)$.

3.4. Time Evolution of $r R 2^{\prime}$ in Different Ischemic Brain Tissue. Respective time evolution of rR2' values in infarcted core, IP, and oligemia was summarized in Table 3 . The infarcted core showed a decreased trend after reperfusion $(P<.05)$ : the maximum decrease happened within the first 6 hours and then maintained as low level as 0 during 6-48 hours. While rR2' values in IP and oligemia showed similar increased 


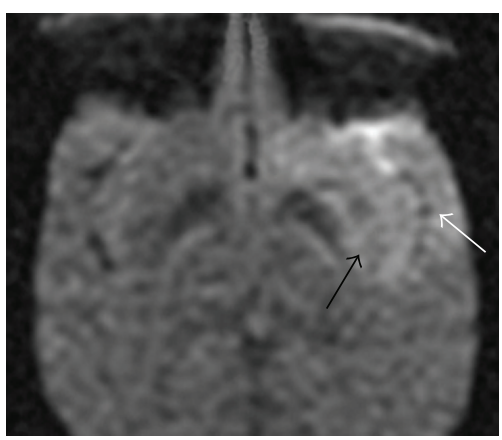

Figure 5: $0 \mathrm{~h}$ DWI shows high-signal ischemic lesion in basal ganglion (black arrow) and temporal lobe (white arrow).

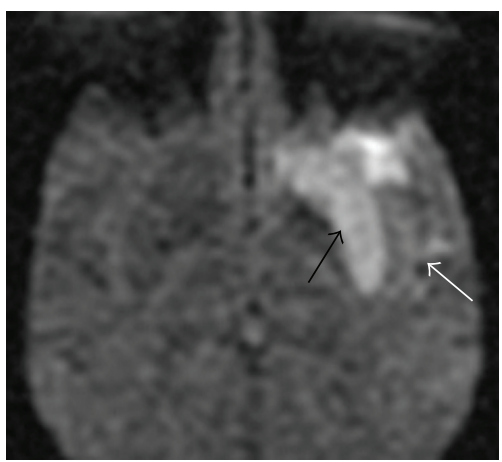

FIGURE 6: $1 \mathrm{~h}$ DWI shows increased signal in basal ganglion (black arrow) but decreased signal in temporal lobe (white arrow).

trend after reperfusion $(P<.05)$ : the maximum increase also happened within the first 6 hours: slightly decreased but still higher than contralateral hemisphere at 1 hour, started to increase at 3 hours, sharply increased at 6 hours, rR2' values maintained a plateau during 6-24 hours and then slightly increased at 48 hours. At each time point, rR2' values in oligemia were slightly higher than those in IP (Figure 12).

\section{Discussion}

4.1. Monkey Model of MCAO. As described by De Crespigny et al. [16], we successfully made monkey model of reversible MCAO by an interventional method in 4/6 monkeys and performed dynamic MRI scans for them. No artifact was found since we removed the microguide wire during MR scan. Unfortunately 2 monkeys died of large area of hemorrhagic infarction in this study. This may due to the fact that we used 250,000 units urokinase to ensure effective reperfusion after the micocratheter was removed.

Although many neuroprotective drugs have proven successful in animal (rodent) models, all have failed during clinical trials. A common reason for this is the lack of demonstrated efficacy of these drugs in larger animals such as cats or monkeys. The subhuman monkeys have similarity in cerebrovascular anatomy, neurochemistry, and immunology, which make them suitable for testing new drug therapies and endovascular instruments [16].

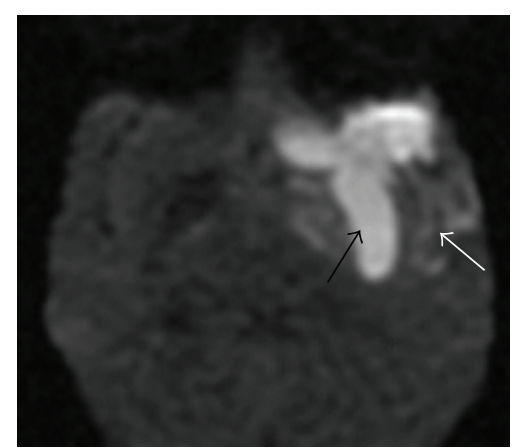

Figure 7: $48 \mathrm{~h}$ DWI shows high-signal in basal ganglion (black arrow) and signal recovery in temporal lobe with scattered high signal (white arrow).

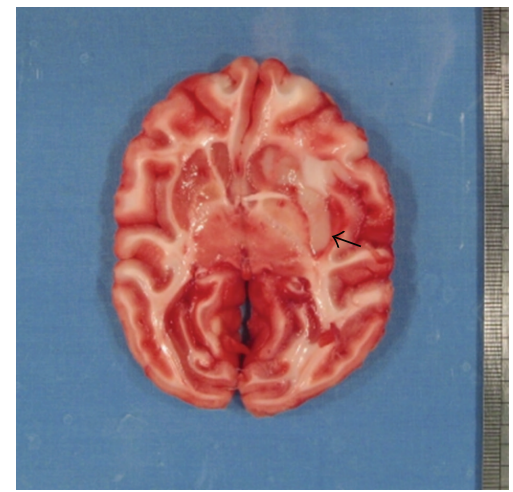

Figure 8: Same animal with Figures 5, 6, and 7, TTC staining shows infarction in basal ganglion (black arrow), temporal lobe did not develop to infarction. The recovered high signal on DWI is IP.

4.2. BOLD-Based R2' MRI in Different Ischemic Brain Tissues. The delineation of the "penumbra" is of particular interest in acute stroke imaging. Since the "mismatch concept" applying PWI and DWI appears to be an oversimplification of the underlying electrophysiological tissue status, an additional parameter reflecting the metabolic state of brain tissue will improve the ability to describe the penumbra [12]. BOLD MR T2' imaging is thought to reflect the response of tissue metabolism to that perfusion deficit with increased local deoxy-Hb used as an indicator of increased OEF. Several studies confirmed the additional values of $\mathrm{T} 2{ }^{\prime}$ map in predicting the real penumbra $[12,14,15]$. In this study, we used R2' to describe changes of local deoxy-Hb. Increased local deoxy-Hb leads to hyperintensity on $\mathrm{R} 2^{\prime}$ images, while decreased local deoxy-Hb leads to hypointensity on R2' images. This makes it easier to visually notice changes of local deoxy-Hb.

Our study demonstrated that the values of $\mathrm{rR}^{\prime}$ were increased in IP and oligemia and decreased in infarcted core with time evolution after reperfusion. Thus, the hyperintensity in R2' images may represent increased deoxy-Hb as an indicator of increased OEF, which can increase in IP and oligemia with ongoing CBF impairment. The infarcted core is unsalvable tissue which has lost the compensative ability of 
TABLE 2: Comparison of rR2' $(\bar{x} \pm s)$ in infarcted core, IP, and oligemia at each time point after reperfusion.

\begin{tabular}{lccccccc}
\hline Subtype & $0 \mathrm{~h}$ & $1 \mathrm{~h}$ & $3 \mathrm{~h}$ & $6 \mathrm{~h}$ & $12 \mathrm{~h}$ & $24 \mathrm{~h}$ & $48 \mathrm{~h}$ \\
\hline Infarcted core & $1.129 \pm 0.108$ & $0.668 \pm 0.082$ & $0.536 \pm 0.075$ & $0.259 \pm 0.050$ & $0.385 \pm 0.054$ & $0.083 \pm 0.026$ & $0.246 \pm 0.058$ \\
IP & $1.329 \pm 0.081$ & $1.237 \pm 0.072$ & $1.453 \pm 0.081$ & $2.435 \pm 0.131$ & $2.447 \pm 0.148$ & $1.968 \pm 0.127$ & $3.694 \pm 0.218$ \\
Oligemia & $1.584 \pm 0.103$ & $1.435 \pm 0.066$ & $1.770 \pm 0.141$ & $2.957 \pm 0.177$ & $3.254 \pm 0.184$ & $3.101 \pm 0.144$ & $4.297 \pm 0.322$ \\
\hline$F$ & 36.19 & 134.09 & 256.30 & 803.25 & 743.74 & 1236.26 & 557.02 \\
$P$ & $<.05$ & $<.05$ & $<.05$ & $<.05$ & $<.05$ & $<.05$ & $<.05$ \\
\hline
\end{tabular}

TABLE 3: Time evolution of rR2' in infarcted core, IP, and oligemia after reperfusion (compared by ANOVA).

\begin{tabular}{|c|c|c|c|c|c|c|c|c|}
\hline \multicolumn{3}{|c|}{$\begin{array}{c}\text { Infracted core } \\
(F=96.56, P<.05)\end{array}$} & \multicolumn{3}{|c|}{$\begin{array}{c}\text { IP } \\
(F=188.23, P<.05)\end{array}$} & \multicolumn{3}{|c|}{$\begin{array}{c}\text { Oligemia } \\
(F=154.05, P<.05)\end{array}$} \\
\hline Reperfusion time & Compared time & $P$ & Reperfusion time & Compared time & $P$ & Reperfusion time & Compared time & $P$ \\
\hline \multirow{6}{*}{$0 \mathrm{~h}$} & $1 \mathrm{~h}$ & $*$ & \multirow{6}{*}{$0 \mathrm{~h}$} & $1 \mathrm{~h}$ & - & \multirow{6}{*}{$0 \mathrm{~h}$} & $1 \mathrm{~h}$ & - \\
\hline & $3 \mathrm{~h}$ & $*$ & & $3 \mathrm{~h}$ & - & & $3 \mathrm{~h}$ & 一 \\
\hline & $6 \mathrm{~h}$ & $*$ & & $6 \mathrm{~h}$ & $*$ & & $6 \mathrm{~h}$ & $*$ \\
\hline & $12 \mathrm{~h}$ & $*$ & & $12 \mathrm{~h}$ & $*$ & & $12 \mathrm{~h}$ & $*$ \\
\hline & $24 \mathrm{~h}$ & * & & $24 \mathrm{~h}$ & $*$ & & $24 \mathrm{~h}$ & $*$ \\
\hline & $48 \mathrm{~h}$ & $*$ & & $48 \mathrm{~h}$ & $*$ & & $48 \mathrm{~h}$ & $*$ \\
\hline \multirow{5}{*}{$1 \mathrm{~h}$} & $3 \mathrm{~h}$ & - & & $3 \mathrm{~h}$ & - & \multirow{5}{*}{$1 \mathrm{~h}$} & $3 \mathrm{~h}$ & - \\
\hline & $6 \mathrm{~h}$ & $*$ & & $6 \mathrm{~h}$ & $*$ & & $6 \mathrm{~h}$ & $*$ \\
\hline & $12 \mathrm{~h}$ & $*$ & $1 \mathrm{~h}$ & $12 \mathrm{~h}$ & $*$ & & $12 \mathrm{~h}$ & $*$ \\
\hline & $24 \mathrm{~h}$ & * & & $24 \mathrm{~h}$ & $*$ & & $24 \mathrm{~h}$ & $*$ \\
\hline & $48 \mathrm{~h}$ & * & & $48 \mathrm{~h}$ & $*$ & & $48 \mathrm{~h}$ & $*$ \\
\hline \multirow{4}{*}{$3 \mathrm{~h}$} & $6 \mathrm{~h}$ & * & \multirow{4}{*}{$3 \mathrm{~h}$} & $6 \mathrm{~h}$ & $*$ & \multirow{4}{*}{$3 \mathrm{~h}$} & $6 \mathrm{~h}$ & $*$ \\
\hline & $12 \mathrm{~h}$ & - & & $12 \mathrm{~h}$ & $*$ & & $12 \mathrm{~h}$ & $*$ \\
\hline & $24 \mathrm{~h}$ & $*$ & & $24 \mathrm{~h}$ & $*$ & & $24 \mathrm{~h}$ & $*$ \\
\hline & $48 \mathrm{~h}$ & $*$ & & $48 \mathrm{~h}$ & $*$ & & $48 \mathrm{~h}$ & $*$ \\
\hline \multirow{3}{*}{$6 \mathrm{~h}$} & $12 \mathrm{~h}$ & - & & $12 \mathrm{~h}$ & - & \multirow{3}{*}{$6 \mathrm{~h}$} & $12 \mathrm{~h}$ & - \\
\hline & $24 \mathrm{~h}$ & - & $6 \mathrm{~h}$ & $24 \mathrm{~h}$ & - & & $24 \mathrm{~h}$ & - \\
\hline & $48 \mathrm{~h}$ & - & & $48 \mathrm{~h}$ & $*$ & & $48 \mathrm{~h}$ & $*$ \\
\hline \multirow{2}{*}{$12 \mathrm{~h}$} & $24 \mathrm{~h}$ & $*$ & \multirow{2}{*}{$12 \mathrm{~h}$} & $24 \mathrm{~h}$ & - & \multirow{2}{*}{$12 \mathrm{~h}$} & $24 \mathrm{~h}$ & - \\
\hline & $48 \mathrm{~h}$ & - & & $48 \mathrm{~h}$ & $*$ & & $48 \mathrm{~h}$ & $*$ \\
\hline $24 \mathrm{~h}$ & $48 \mathrm{~h}$ & - & $24 \mathrm{~h}$ & $48 \mathrm{~h}$ & $*$ & $24 \mathrm{~h}$ & $48 \mathrm{~h}$ & $*$ \\
\hline
\end{tabular}

${ }^{*} P<.05,-P>.05$.

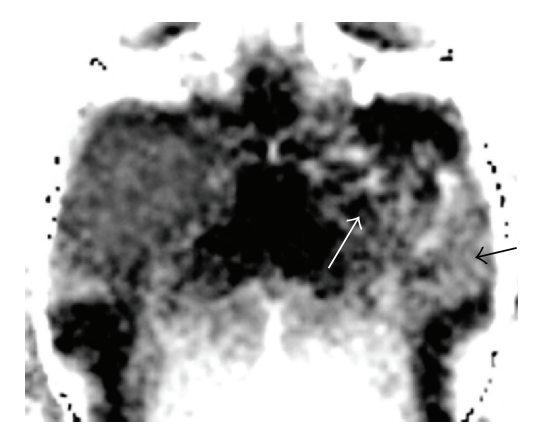

FIGURE 9: $0 \mathrm{~h} \mathrm{R} 2^{\prime}$ map shows that the signal of IP (black arrow) is higher than that of infarcted core (white arrow).

increased OEF. Thus, the infarcted core demonstrated consecutive hypointensity in $\mathrm{R} 2$ ' images even after reperfusion. PET study has showed 50\% increasement of OEF in IP [18].
An interesting finding demonstrated by Geisler et al. [12] was the infarcted core showed even shorter $\mathrm{T} 2{ }^{\prime}$ than lesion growth (operationally defined IP, $-15.7 \%$ versus $-10.5 \%$ ) although there was no statisticlly significant difference. That result was not as they have expected: a strongly reduced $\mathrm{O}_{2}$ utilization, a clearly lowered OEF, a decreased deoxy-Hb, and consecutively increased $\mathrm{T} 2$ ' in infarcted core. That result was also inconsistent with the widely accepted concept of IP $[19,20]$ and with our results. This may be due to the fact that they defined infarcted core by decreased ADC within 6 hours after symptom onset. This reinforced the concept that ADC lesion may not only describe the infarcted core but also include a considerable portion of IP which showed decreased $\mathrm{T}^{\prime}$. Furthermore, previously produced deoxy- $\mathrm{Hb}$ cannot effectively be removed from the highly reduced CBF and may thus account for the T2' shortening [12].

Although both IP and oligemia showed similar increased trend in this study, values of $r R 2^{\prime}$ in oligemia were higher 


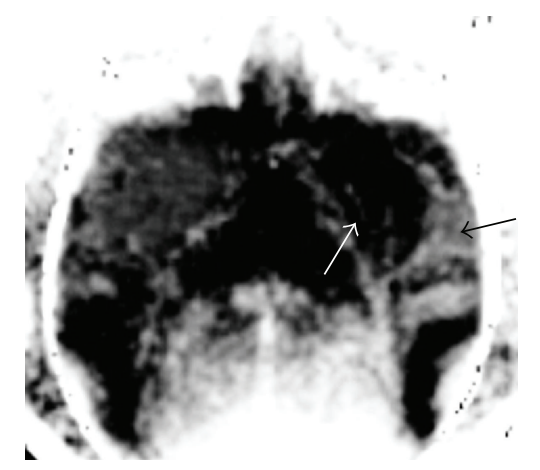

Figure 10: $1 \mathrm{~h} \mathrm{R} 2$ ' map shows low signal of irreversible infarcted core (white arrow), slightly high signal of reversible IP (black arrow).

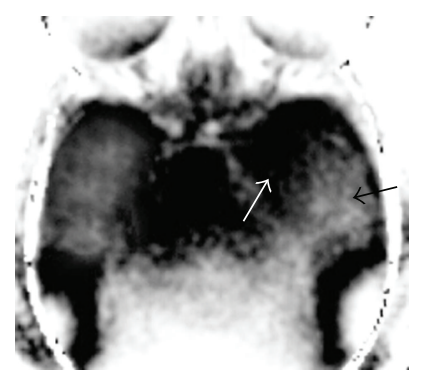

FIGURE 11: $48 \mathrm{~h} \mathrm{R} 2$ ' map shows significant low signal of infarcted core (white arrow), high signal of IP (black arrow) (Figures 9, 10, and 11 is same animal as Figures 5, 6, and 7).

than that in IP at every time point after reperfusion. There are two reasons for this. Firstly, CBF in oligemia is less impaired than those in IP [21], which means that the oxygen supply in oligemia is more sufficient. Secondly, the compensative ability of oligemia is stronger than that of IP, which leads to more increased $\mathrm{OEF}$ and increased generation of deoxy-Hb. The less decreased CBF and more increased $\mathrm{OEF}$ are the real reasons that oligemia can escape from ischemic injury and never develop to infarction. Certainly, we can establish a rR2' threshold to distinguish IP and oligemia. Considering the limited number of our animal models, we would leave this job for further study.

4.3. Time Evolution of $r R 2^{\prime}$ in Different Ischemic Brain Tissues. Both PET and BOLD MRI studies had showed increased OEF in acute ischemic stroke $[4-6,12,14,15]$. But the duration of increased OEF is still unknown. According to the results of our study, time evolution of rR2 in IP and oligemia can be classified into 3 stages: sharply increased within first 6 hours, maintained a high level plateau during 6-24 hours, and then slightly increased until 48 hours. While the infarcted core had 2 stages: sharply decreased within first 6 hours and maintained as low level as 0 from 6 to 48 hours. 6 hours is also the time window for venous thrombolytic therapy in acute ischemic stroke and is widely used in many stroke studies $[1,4,6,12,14,18,22]$. Early recanalization is the first and most important step to save IP. The most obvious

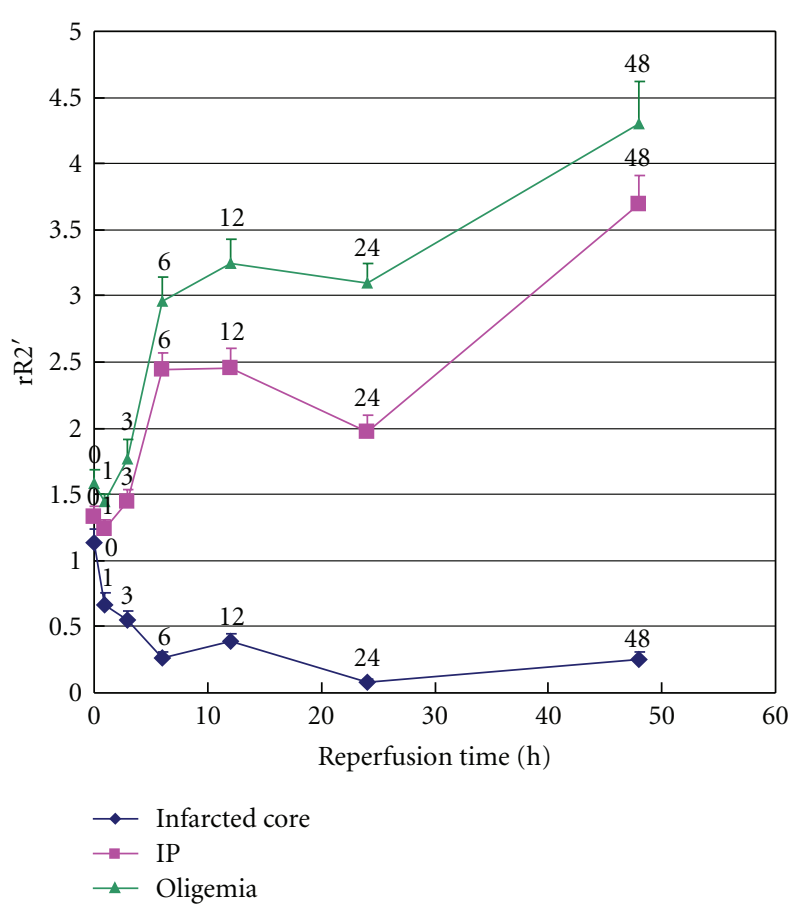

FIGURE 12: Reperfusion time evolution of rR2' shows decreased trend in infarcted core but increased trend in IP and oligemia.

hemodynamic changes may happen during the first 6 hours after early reperfusion [21]. We presume quick recovered $\mathrm{CBF}$ may encourage IP to increase OEF excessively, generate more deoxy-Hb, and lead to sharply increased rR2'. With the gradual recovery of CBF from 6 to 24 hours, IP may still be in moderate anoxic status and OEF may still increase at a lower speed. The plateau during 6-24 hours may represent the balance state between recovering CBF and elevated OEF. At 48 hours CBF may have already completely recovered but OEF may still be maintained at a higher level, which leads to slightly increased rR2'. That is to say, increased OEF may last at least 48 hours after early reperfusion. A previous study confirmed that extended duration of increased OEF will benefit brain tissue at risk [22]. 48 hours duration of increased deoxy-Hb disclosed by our study may be helpful to extend therapy time window of ischemic stroke.

For the infarcted core, the sharply decreased rR2' within the first 6 hours may be due to the fact that no generation of deoxy-Hb is found even after early reperfusion. With the quick recovery of $\mathrm{CBF}$, the previously produced deoxy- $\mathrm{Hb}$ is removed and rR2' maintains as low level as 0 after the first 6 hours.

There are some methodological limitations for $\mathrm{T} 2^{\prime}$ or R2' images. Firstly, T2* is sensitive to large variations of the static magnetic field $(\Delta \mathrm{B} 0)$. This may affect the accuracy of measured $\mathrm{T} 2$ ' or $\mathrm{R} 2{ }^{\prime}$. This problem can be solved by using side-to-side comparison which reveals more valuable relative results. Secondly, the concentration of deoxy-Hb in a single voxel is determined mainly by 2 factors: changes in the local generation of deoxy-Hb inside the voxel and changes in the transport of deoxy-Hb into or out of the voxel, 
which is closely correlated with CBF [12]. So information of CBF should be imposed on $\mathrm{T} 2$ ' or R2' images. A study carried by Jensen et al. [15] showed that there was a moderate correlation between rCBF derived from PWI and qT2'. qT2' maps could be used to explore cerebral perfusion without the application of contrast agent or radiation.

Although BOLD-based MR quantitative OEF measurement has been published and demonstrated good correlation with the existing OEF values $[23,24]$. The clinical value of this technology is still unknown. Further studies should be focused on the improvement of accuracy of cerebral oxygen extraction by using BOLD MR.

\section{Conclusions}

BOLD-based R2' MRI can be used to describe changes of cerebral oxygen extract in acute ischemic stroke, and it can provide additional information in detecting IP. The time evolution rR2' in infarcted core, IP, and oligemia is in accordance with the underlying pathophysiolgy. With improvement in the accuracy, this technique would add more valuable information for patients selection for thrombolytic therapy.

\section{Acknowledgments}

This study was partially supported by Tianjin Municipal Nature Science Foundation (no. 09JCYBJC11500), National Basic Research Program of China (973 program, no. 2010CB732506), National Natural Science Foundation of China (NSFC, no. 30730036), and Doctorial Foundation of Ministry of Education of China (no. 20091202110006).

\section{References}

[1] J. Röther, P. D. Schellinger, A. Gass et al., "Effect of intravenous thrombolysis on MRI parameters and functional outcome in acute stroke <6 hours," Stroke, vol. 33, no. 10, pp. 2438-2445, 2002.

[2] P. A. Barber, M. W. Parsons, P. M. Desmond et al., "The use of PWI and DWI measures in the design of "proof-of-concept" stroke trials," Journal of Neuroimaging, vol. 14, no. 2, pp. 123132,2004

[3] P. D. Schellinger, J. B. Fiebach, and W. Hacke, "Imaging-based decision making in thrombolytic therapy for ischemic stroke: present status," Stroke, vol. 34, no. 2, pp. 575-582, 2003.

[4] C. S. Kidwell, J. R. Alger, and J. L. Saver, "Beyond mismatch: evolving paradigms in imaging the ischemic penumbra with multimodal magnetic resonance imaging," Stroke, vol. 34, no. 11, pp. 2729-2735, 2003.

[5] J. Fiehler, K. Knudsen, T. Kucinski et al., "Predictors of apparent diffusion coefficient normalization in stroke patients," Stroke, vol. 35, no. 2, pp. 514-519, 2004.

[6] C. B. Grandin, T. P. Duprez, A. M. Smith et al., "Which MRderived perfusion parameters are the best predictors of infarct growth in hyperacute stroke? Comparative study between relative and quantitative measurements," Radiology, vol. 223, no. 2, pp. 361-370, 2002.

[7] H. Tamura, J. Hatazawa, H. Toyoshima, E. Shimosegawa, and T. Okudera, "Detection of deoxygenation-related signal change in acute ischemic stroke patients by $\mathrm{T} 2{ }^{*}$-weighted magnetic resonance imaging," Stroke, vol. 33, no. 4, pp. 967971, 2002.

[8] S. A. Roussel, N. Van Bruggen, M. D. King, and D. G. Gadian, "Identification of collaterally perfused areas following focal cerebral ischemia in the rat by comparison of gradient echo and diffusion-weighted MRI," Journal of Cerebral Blood Flow and Metabolism, vol. 15, no. 4, pp. 578-586, 1995.

[9] J. M. Wardlaw and A. Von Heijne, "Increased oxygen extraction demonstrated on gradient echo (T2*) imaging in a patient with acute ischaemic stroke," Cerebrovascular Diseases, vol. 22, no. 5-6, pp. 456-458, 2006.

[10] N. Morita, M. Harada, M. Uno et al., "Ischemic findings of T* weighted 3-tesla MRI in acute stroke patients," Cerebrovascular Diseases, vol. 26, no. 4, pp. 367-375, 2008.

[11] M. L. Donswijk, P. S. Jones, J. V. Guadagno et al., “T2*weighted MRI versus oxygen extraction fraction PET in acute stroke," Cerebrovascular Diseases, vol. 28, no. 3, pp. 306-313, 2009.

[12] B. S. Geisler, F. Brandhoff, J. Fiehler et al., "Blood oxygen leveldependent MRI allows metabolic description of tissue at risk in acute stroke patients," Stroke, vol. 37, no. 7, pp. 1778-1784, 2006.

[13] H. An and W. Lin, "Quantitative measurements of cerebral blood oxygen saturation using magnetic resonance imaging," Journal of Cerebral Blood Flow and Metabolism, vol. 20, no. 8, pp. 1225-1236, 2000.

[14] S. Siemonsen, T. Fitting, G. Thomalla et al., "T2' imaging predicts infarct growth beyond the acute diffusion-weighted imaging lesion in acute stroke," Radiology, vol. 248, no. 3, pp. 979-986, 2008.

[15] U. R. Jensen, J.-R. Liu, C. Eschenfelder et al., "The correlation between quantitative $\mathrm{T} 2$ ' and regional cerebral blood flow after acute brain ischemia in early reperfusion as demonstrated in a middle cerebral artery occlusion/reperfusion model of the rat," Journal of Neuroscience Methods, vol. 178, no. 1, pp. 55-58, 2009.

[16] A. J. De Crespigny, H. E. D’Arceuil, K. I. Maynard et al., “Acute studies of a new primate model of reversible middle cerebral artery occlusion," Journal of Stroke and Cerebrovascular Diseases, vol. 14, no. 2, pp. 80-87, 2005.

[17] T. Rohlfing, C. R. Maurer, D. A. Bluemke, and M. A. Jacobs, "Volume-preserving nonrigid registration of MR breast images using free-form deformation with an incompressibility constraint," IEEE Transactions on Medical Imaging, vol. 22, no. 6, pp. 730-741, 2003.

[18] J. Sobesky, O. Zaro Weber, F. G. Lehnhardt et al., "Does the mismatch match the penumbra? Magnetic resonance imaging and positron emission tomography in early ischemic stroke," Stroke, vol. 36, no. 5, pp. 980-985, 2005.

[19] W. D. Heiss, "Ischemic penumbra: evidence from functional imaging in man," Journal of Cerebral Blood Flow and Metabolism, vol. 20, no. 9, pp. 1276-1293, 2000.

[20] M. Kavec, O. H. J. Gröhn, M. I. Kettunen, M. J. Silvennoinen, M. Penttonen, and R. A. Kauppinen, "Use of spin echo T BOLD in assessment of cerebral misery perfusion at 1.5 T," Magnetic Resonance Materials in Physics, Biology and Medicine, vol. 12, no. 1, pp. 32-39, 2001.

[21] E. Bandera, M. Botteri, C. Minelli, A. Sutton, K. R. Abrams, and N. Latronico, "Cerebral blood flow threshold of ischemic penumbra and infarct core in acute ischemic stroke: a systematic review," Stroke, vol. 37, no. 5, pp. 1334-1339, 2006. 
[22] J. V. Guadagno, E. A. Warburton, F. I. Aigbirhio et al., "Does the acute diffusion-weighted imaging lesion represent penumbra as well as core? A combined quantitative PET/MRI voxelbased study," Journal of Cerebral Blood Flow and Metabolism, vol. 24, no. 11, pp. 1249-1254, 2004.

[23] X. He and D. A. Yablonskiy, "Quantitative BOLD: mapping of human cerebral deoxygenated blood volume and oxygen extraction fraction: default state," Magnetic Resonance in Medicine, vol. 57, no. 1, pp. 115-126, 2007.

[24] X. He, M. Zhu, and D. A. Yablonskiy, "Validation of oxygen extraction fraction measurement by qBOLD technique," Magnetic Resonance in Medicine, vol. 60, no. 4, pp. 882-888, 2008. 


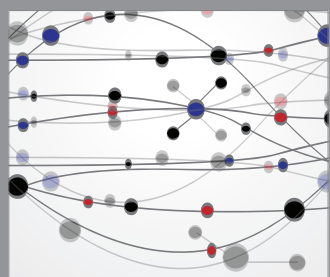

The Scientific World Journal
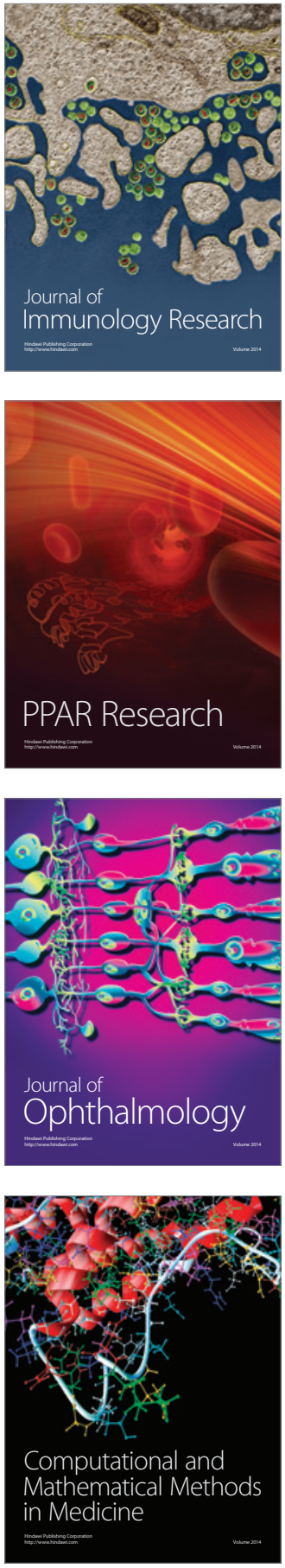

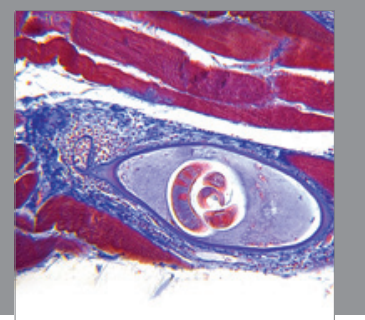

Gastroenterology

Research and Practice
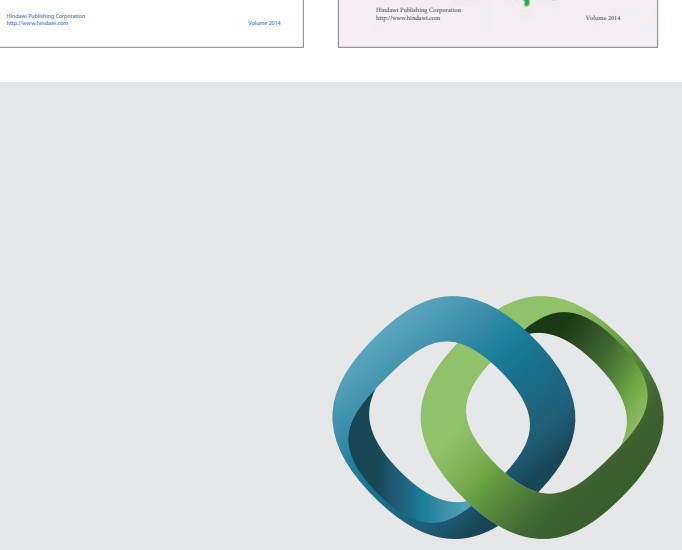

\section{Hindawi}

Submit your manuscripts at

http://www.hindawi.com
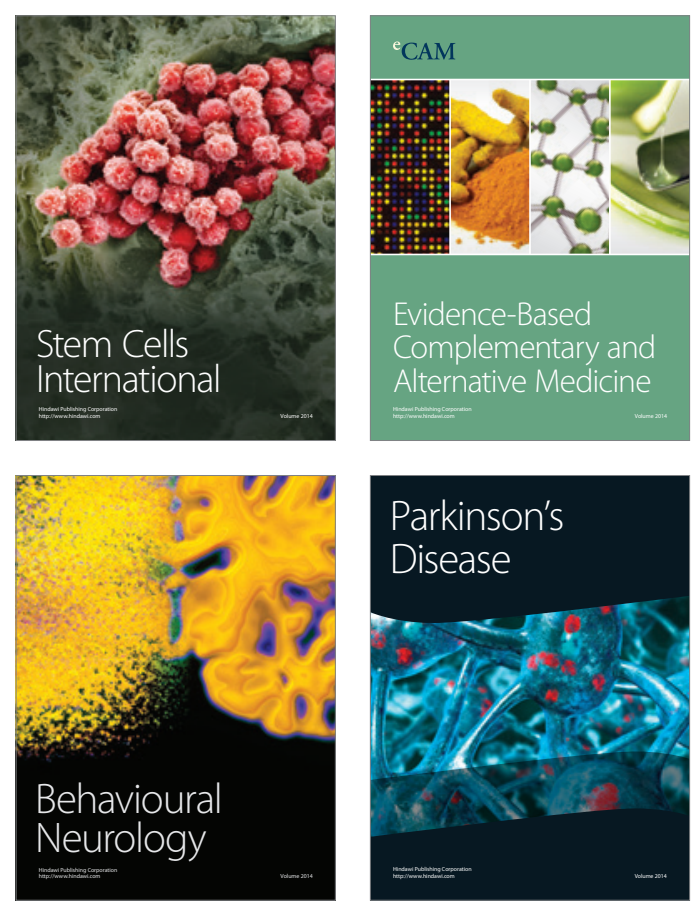

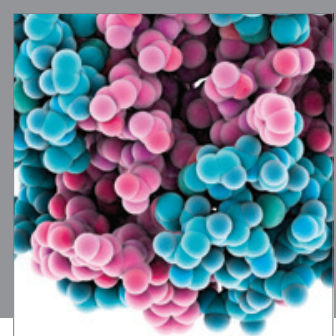

Journal of
Diabetes Research

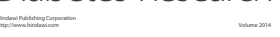

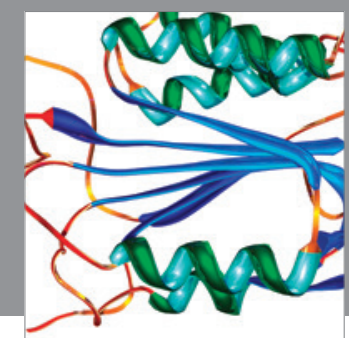

Disease Markers
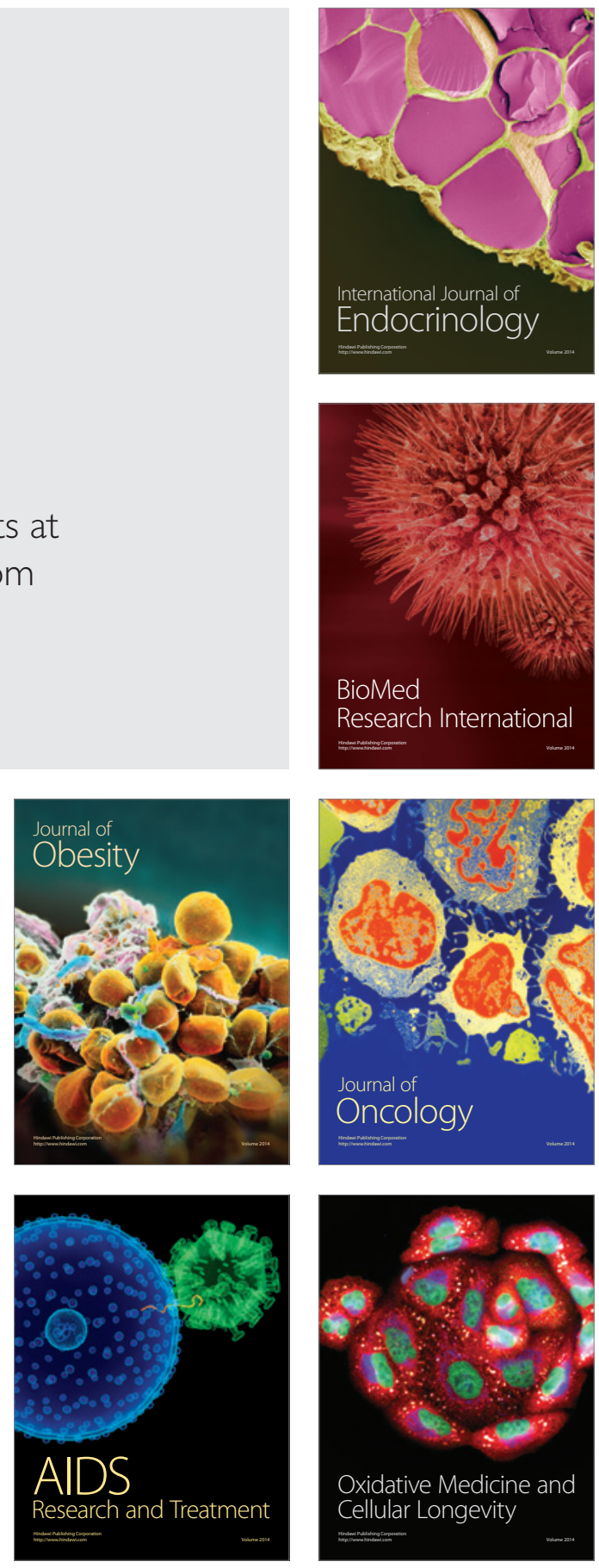